\title{
Target Sample Size
}

National Cancer Institute

\section{Source}

National Cancer Institute. Target Sample Size. NCI Thesaurus. Code C139172.

The total number of planned participants in a study or trial. 